\title{
Animais peçonhentos em rede: uma exposição multi-museus
}

\author{
Rejâne M. Lira-da-Silva' \\ Giselle Agostini Gotta ${ }^{2}$ \\ Giuseppe Puorto 3 \\ Felipe Barbosa Dias ${ }^{4}$ \\ Micheli Ferreira Fonsêca ${ }^{5}$ \\ Ivson Santos Gomes ${ }^{6}$ \\ Érico Vital Brazil', \\ Tania Kobler Brazil ${ }^{8}$
}

\section{Resumo}

A divulgação sobre animais peçonhentos é um desafio que pressupõe conceitos científicos equivocados, crenças populares e dificuldade ao acesso à informação. Relatamos a experiência sobre a concepção, criação e execução da Exposição “Animais Peçonhentos em Rede" no $2^{\circ}$ Encontro Internacional Vital para o Brasil, em Campanha/Minas Gerais, que recebeu milhares de visitantes e trouxe o caráter inovador de aproximar e promover a interação entre público e cientistas, em uma atmosfera lúdica, dada a similitude da práxis museológica das instituições: a educação sobre animais peçonhentos. Ações de educação museal multi-museus são importantes quando integram espaços expositivos com múltiplas estratégias pedagógicas que suscitem reflexões e ações da sociedade.

\section{Palavras-chave:}

Animais peçonhentos; Educação museal; Museus.

\begin{abstract}
The disclosure about venomous animals is a challenge that assumes erroneous scientific concepts, popular beliefs and difficulty accessing information. We report on the design, creation and execution of the "Poisonous Animals in Network" Exhibition at the 2nd Vital International Meeting for Brazil, in Campanha (Minas Gerais), which received thousands of visitors and brought the innovative character of approaching and promoting interaction between public and scientists, given the similarity of the museological praxis of institutions: education on venomous animals. Actions of the museum education are important when they integrate exhibition spaces with multiple pedagogical strategies that give rise to reflections and actions.
\end{abstract}

\section{Keywords:}

Venomous animals; Museum education; Museums.

I Núcleo de Ofiologia e Animais Peçonhentos da Bahia, Universidade Federal da Bahia, Salvador, Bahia, Brasil.rejane@ufba.br.

2 Fundação Ezequiel Dias, Belo Horizonte, Minas Gerais, Brasil. gacotta@gmail.com

3 Instituto Butantan, São Paulo, Bahia, Brasil.g.puorto@gmail.com.

4 Núcleo de Ofiologia e Animais Peçonhentos da Bahia, Universidade Federal da Bahia, Salvador, Bahia, Brasil

5 Núcleo de Ofiologia e Animais Peçonhentos da Bahia, Universidade Federal da Bahia, Salvador, Bahia, Brasil

6 Núcleo de Ofiologia e Animais Peçonhentos da Bahia, Universidade Federal da Bahia, Salvador, Bahia, Brasil

7 InstitutoVital Brazil, Niterói, Rio de Janeiro, Brasil. ericovitalbrazil@gmail.com. Casa deVital Brazil, Campanha, Minas Gerais, Brasil.

8 Núcleo de Ofiologia e Animais Peçonhentos da Bahia, Universidade Federal da Bahia, Salvador, Bahia, Brasil 
Introdução

Atualmente, a dimensão educacional vem se ampliando nos museus de ciências com a incorporação das novas tecnologias de comunicação. Os conceitos científicos, ideias e objetos, ao serem expostos, passam por transformações que implicam na simplificação, reorganização e produção de novos conhecimentos, necessários para levar o público a compreender a ciência apresentada nos museus. Esse processo ocorre por variadas razões: valorização do visitante e necessidade de promover ações que garantam qualidade do conhecimento científico divulgado nas exposições. Os museus devem não só fazer suas exposições e contar as histórias dos objetos das coleções, como desenvolver atividades educativas e culturais que revelem não somente os conceitos envolvidos, mas também o processo de produção de conhecimentos com base nos objetos. As exposições são estratégias para o desenvolvimento de atividades educativas nos museus. Por meio delas é possível realizar comparações entre seres e ambientes, compreendendo suas relações, estudar comportamentos, entender como os espécimes são coletados, conservados, pesquisados e classificados, acessando as informações de caráter teórico e procedimental das Ciências Naturais. É possível também apresentar a dimensão processual da ciência, fornecendo aos visitantes informações sobre as controvérsias que caracterizam a produção do conhecimento científico (MARANDINO, 20I4).

Educar sobre animais peçonhentos no Brasil é um desafio. Esta fauna, em específico, está associada a diversas crenças populares, geralmente negativas, que acabam por dificultar a comunicação com o público em geral. $\mathrm{Na}$ visão de estudantes, por exemplo, o senso comum é um tipo de conhecimento predominante, que sobrevive e convive associado ao perfil conceitual deste grupo, mesmo após o ensino de noções científicas (SANTOS; LIRA-DA-SILVA, 20I2).

A escassez no mercado de profissionais com formação técnica e científica também se constitui como uma barreira no acesso a informação sobre animais peçonhentos. Dentro do espectro da educação, a ausência deste tipo de profissionais, como professores e divulgadores de ciência, aparenta ser ainda maior - o que dificulta ainda mais o processo - visto que poucas são as instituições brasileiras que se comprometem com o caráter divulgativo do conhecimento científico produzido. Tal problema provavelmente deriva da baixa oferta de disciplinas, que em seu conteúdo programático discutam sobre os animais peçonhentos. Nos cursos de Ciências Biológicas, por exemplo, em instituições federais de ensino superior (IFES) de ensino, apenas 17 (38\%) das 45 IFES analisadas possuem em seus currículos, conteúdos que tratem sobre este tema, disponibilizados em maioria como componentes optativos ou eletivos (ARANHA; LIRA-DA-SILVA, 20I3).

A Rede Nacional de Informação, Diálogo e Cooperação Acerca dos Animais Peçonhentos - Rede Vital para o Brasil (RVB) foi criada em 2010 com o objetivo principal a promoção do diálogo e da cooperação entre as diversas instituições e profissionais que estudam os animais peçonhentos e seus venenos. A RedeVital para o Brasil se constitui como um esforço conjunto entre a Fundação Ezequiel Dias (FUNED), a Casa de Vital Brazil (CVB), o Instituto Butantan (IB), o Instituto Vital Brazil (IVB) e Núcleo de Ofiologia e Animais Peçonhentos da Universidade Federal da Bahia (NOAP/UFBA), para fornecer suporte permanente ao intercâmbio de informações e experiências, ao estímulo para a formação qualificada, para a preservação da memória e para a divulgação de conteúdo científico. $A$ Rede Vital para o Brasil promove eventos científicos voltados para divulgação 
dos animais peçonhentos e suas interfaces, além de encontros sobre coleções (BRAZIL, 20I I; BRAZIL et al., 20I2).

No ano de 20I4, entre os dias II e I4 de novembro, a RVB realizou na cidade de Campanha, Minas Gerais, município que nasceu o Cientista Vital Brazil, - $2^{\circ}$ Encontro Internacional Vital para o Brasil (EIVB) sobre animais peçonhentos cujo tema foi: Os I 20 anos da Soroterapia antiveneno - dos soros à bioprospecção. O objetivo deste encontro foi promover a aproximação de pesquisadores, estudantes e instituições a fim de estimular a cooperação permanente e as ações conjuntas em prol da qualificação profissional, do avanço de pesquisas e do melhor servir e divulgar informações preventivas a todas as comunidades que vivem em risco de acidentes com animais peçonhentos. Além dos minicursos, mesas redondas e palestras que compuseram o encontro, a exposição "Animais Peçonhentos em Rede" integrou a programação do $2^{\circ}$ EIVB.

A exposição foi feita no contexto de rede, que se refere a um conjunto de ações características de um espaço social (ANDRADE, 2010). A RVB é uma dessas redes, no contexto sócio-institucional que inclui museus representados, com atividades e ações relativas à comunicação pública sobre os animais peçonhentos.

O objetivo deste artigo é apresentar um relato de experiência sobre a criação e execução da exposição “Animais Peçonhentos em Rede", que envolveu um esforço conjunto de cinco museus brasileiros em atividades educacionais voltadas para diferentes públicos.

O presente trabalho teve uma abordagem de pesquisa qualitativa, tratando-se de um relato de experiência das atividades realizadas na exposição "Animais Peçonhentos em Rede" durante o $2^{\circ}$ EIVB, organizada por 5 museus cujo eixo articulador foi o ensino, a pesquisa e a extensão sobre esses animais.

\section{A exposição "Animais Peçonhentos em Rede"}

A exposição foi uma alusão à comunicação científica sobre animais peçonhentos por instituições museais, aberta à toda a sociedade da cidade de Campanha, particularmente à comunidade escolar da educação básica ao ensino superior.

A musealização da exposição teve como objetivos partilhar as múltiplas experiências para a educação científica sobre animais peçonhentos de 5 Espaços Museais, FUNED (Belo Horizonte, MG), IVB (Niterói, RJ), Museu Biológico do IB (São Paulo, SP) e NOAP/UFBA (Salvador, BA) (Tabela I), valorizando-os como espaços interdisciplinares e de grande importância regional e nacional; reunir pela primeira vez em um encontro sobre animais peçonhentos, instituições e profissionais diretamente relacionadas com a produção e divulgação do conhecimento científico sobre a fauna brasileira de animais peçonhentos; valorizar essas instituições com a divulgação das suas pesquisas, visando a inclusão científica e o despertar de vocações científicas de futuros zoólogos e áreas afins; discutir - papel desses museus como patrimônio material nacional, indispensável para o desenvolvimento científico e tecnológico e para o desenvolvimento de ações conservacionistas da fauna de animais peçonhentos no Brasil. 
Tabela I. Instituições que participaram da exposição "Animais Peçonhentos em Rede"

\begin{tabular}{|l|l|l|l|}
\hline \multicolumn{1}{|c|}{ Instituição } & Cidade de Origem & Pesquisador Responsável & Ferramentas Educativas \\
\hline Fundação Ezequiel Dias & Belo Horizonte, MG & Giselle A. Costa & Ciência em Movimento \\
\hline Instituto Butantan & São Paulo, SP & Giuseppe Porto & Réplicas de serpentes \\
\hline Instituto Vital Brazil & Niterói, RJ & Érico T.Vital Brazil & Animais Vivos \\
\hline $\begin{array}{l}\text { Núcleo de Ofiologia e Ani- } \\
\text { mais Peçonhentos UFBA/ } \\
\text { NOAP }\end{array}$ & Salvador, BA & Rejane M. Lira-da-Silva & $\begin{array}{l}\text { REDEZOO em Cena e Lu- } \\
\text { doteca }\end{array}$ \\
\hline Casa de Vital Brazil (CVB) & Campanha, MG & Érico T.Vital Brazil & Fotos, cartas, etc.. \\
\hline
\end{tabular}

\section{O plano museológico da exposição}

Quando a RVB estabeleceu parceria entre diversas instituições que estudam sobre animais peçonhentos também se notou a necessidade da divulgação científica, em rede, desses animais. Em 2013, a RVB realizou sua primeira exposição, “Ninho dos Cobras”, noVI Congresso Brasileiro de Herpetologia. Tal exposição se constituiu como um projeto que consolidaria as ações educativas da RVB e foi uma experiência exitosa que estimulou a exposição "Animais Peçonhentos em Rede" (Figura I).

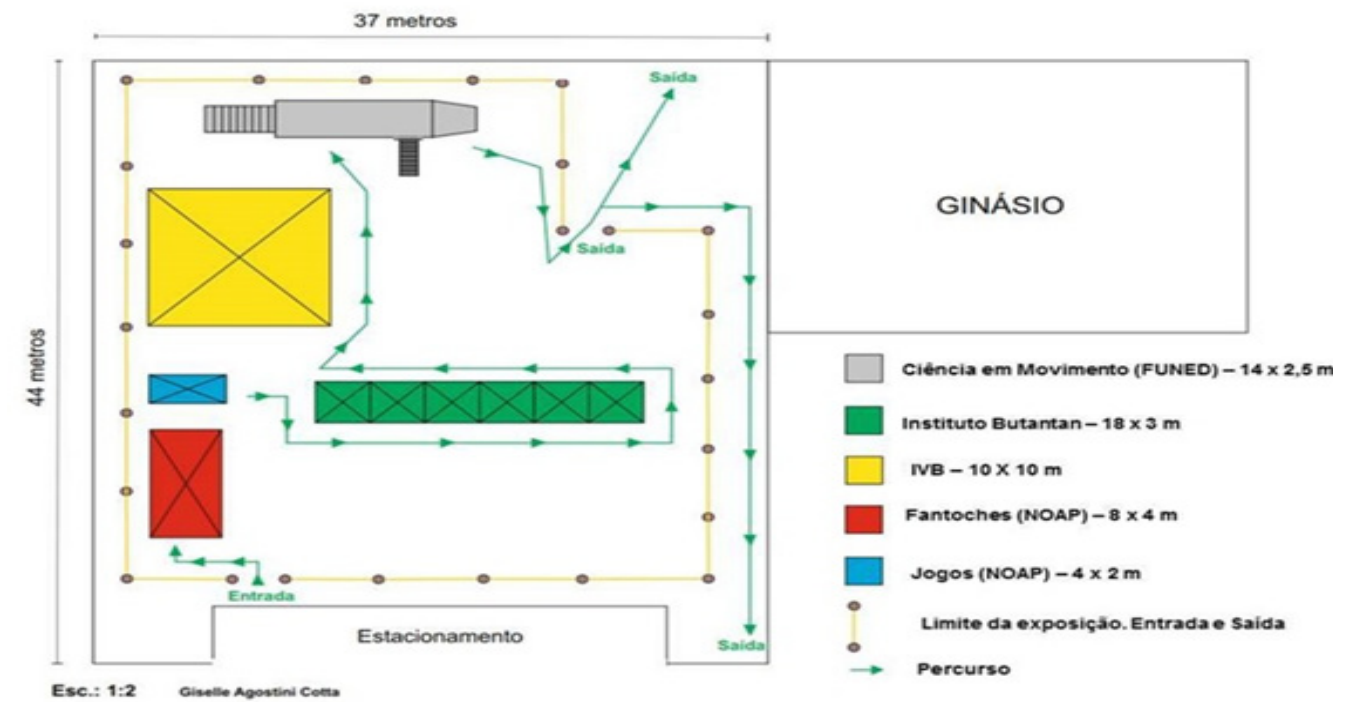

Figura I: Plano museográfico da exposição “Animais Peçonhentos em Rede”, Campanha, Minas Gerais.

As ações educativas do NOAP/UFBA fazem parte do projeto de extensão universitária Rede de Zoologia Interativa (REDEZOO), um programa de produção de conhecimento e popularização da Zoologia, através de exposições itinerantes.As ferramentas deste programa utilizadas na exposição "Animais Peçonhentos em Rede" foram a REDEZOO em cena, por meio do uso do Teatro de Fantoches, e a Zooteca e Zookits, com experimentos e jogos lúdicos voltados para o ensino e divulgação dos animais peçonhentos. $O$ uso do Teatro de Fantoches como instrumento educativo na divulgação científica foi conduzido em consonância com o trabalho de Lira-da-Silva et al (2007). Foram adaptadas para o público de Campanha as peças "Carlinhos e a Cascavel” (PINTO-COE- 
LHO, 20I0) e "O Lixo é a casa do Bicho" (LIRA-DA-SILVA, 20I I).

A FUNED trouxe à exposição o projeto "Ciência em Movimento", formada a partir da experiência da instituição com animais peçonhentos, especialmente as serpentes. Criado em 2012, o "Ciência em Movimento" tinha como objetivo difundir o conhecimento científico e tecnológico, através de linguagem lúdica e popular, estabelecendo uma relação de diálogo entre os pesquisadores e a sociedade e entre ciência, saúde e cultura. $O$ programa realiza trabalhos de popularização da ciência em diferentes municípios de Minas Gerais. Os visitantes têm acesso a exposições sobre animais peçonhentos (morfologia e diversidade animal), produção de medicamentos e soros, vigilância sanitária e epidemiológica, entre outros (FUNED, 20I4).

O Instituto Butantan (IB) apresentou a coleção de réplicas de serpentes peçonhentas e não peçonhentas em epox, que faz parte do acervo da instituição, além de itens da coleção pessoal de Giuseppe Puorto, diretor do IB. Esta coleção reproduz os animais o mais fielmente possível preservando suas características, tornando-os elementos importantes para divulgação do conhecimento científico. Além disso, o material é de fácil conservação e permite observar estruturas morfológicas e anatômicas com detalhes sem oferecer risco à manipulação (PUORTO, 20I4).

O IVB expôs sua coleção viva de animais peçonhentos de importância médica, composta por dioramas, caracterizados por serpentes, aranhas e escorpiões em terrários, representando seu ambiente natural, ambientados com troncos, plantas, rochas naturais ou artificiais, cascalhos, areia e bebedouros para garantir o bem-estar dos animais. As espécies expostas foram as mais comuns que ocorrem no país (Bothrops sp - jararaca, Crotalus durissus - cascavel; Boa constrictor - jiboia; Lasiodora sp - aranha caranguejeira; Loxosceles sp - aranha marrom; e Phoneutria sp - aranha armadeira) (REIS et al., 20I4).

A Casa de Vital Brazil (CVB), tombada pelo Instituto do Patrimônio Histórico e Artístico Nacional (IPHAN) em 1985, participou do circuito de visita da exposição. A CVB possui um valioso acervo de fotografias, cartas, documentos, trabalhos científicos, livros e objetos que foram doados pelos familiares de Vital Brazil. Desde sua fundação, o Museu teve como objetivo preservar a memória de Vital Brazil, atuando como pólo de difusão cultural por meio da valorização do resgate histórico e social da cidade de Campanha e tornando-se pólo incentivador da Educação Ambiental, especialmente em temas relacionados à obra de Vital Brazil.

A exposição e o público

A ação foi realizada no Parque de Exposições de Campanha e a mediação da exposição contou com profissionais das 5 instituições na divulgação do tema "animais peçonhentos", onde apresentaram seus conhecimentos sobre estes animais, em relação a biologia, mitos e os acidentes que representam um importante problema de saúde pública.

O público total de visitantes da exposição "Animais Peçonhentos em Rede" foi em torno de 600 pessoas, constituída principalmente por estudantes e professores da rede básica de ensino, universitários e pesquisadores participantes do $2^{\circ}$ EIVB e moradores da cidade de Campanha e região metropolitana (Figura 2). Com tantas pessoas, nosso maior desafio foi o tempo. Para Hein (2009), o tempo é crucial para a aprendizagem e foi por isso que foram utilizadas tantas ferramentas educativas para motivar os visitantes, particularmente as 
crianças.

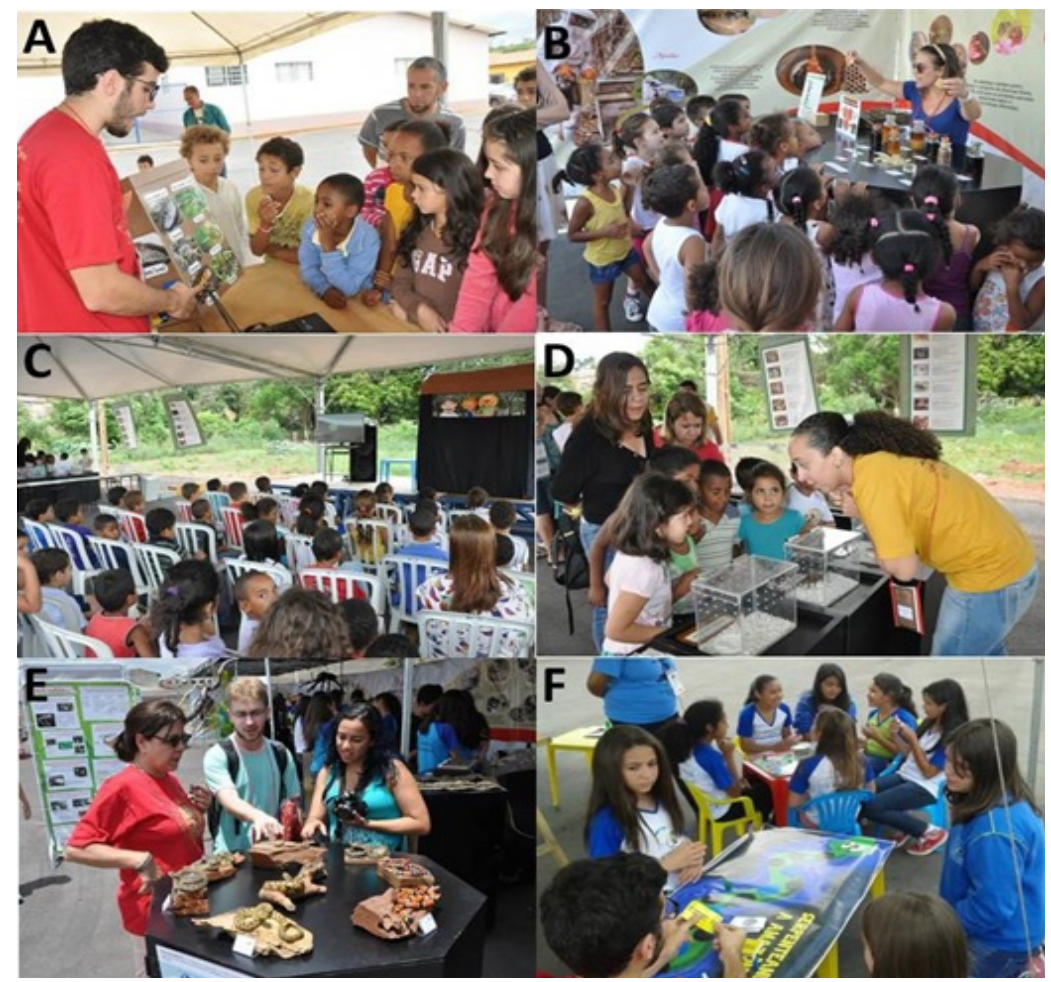

Figura 2: Ações educativas durante a exposição. (A) Experimento "Como as serpentes enxergam no escuro?" Zooteca - NOAP/UFBA. (B) Ciência e Movimento - FUNED. (C) Teatro de Fantoches - NOAP/UFBA. (D) Animais vivos (IVB). (E) Réplicas - IB. (F) Jogo "Serpenteando a Amazônia" Zooteca - NOAP/UFBA.

Uma das preocupações centrais dos divulgadores de ciência é promover uma exposição que, de fato, possibilite uma real e significativa compreensão por seus visitantes. Segundo Marandino (2008), nas últimas décadas houve uma mudança de paradigma frente as abordagens comunicativas em museus, agora assumindo o público como protagonista no processo comunicativo. A mesma autora relata que a responsabilidade em produzir exposições e atividades que contemplem o ator principal, ou seja, os visitantes, pertence aos museus. Sendo assim, é essencial que o visitante seja protagonista da sua própria aprendizagem dentro destes espaços para que a mediação possa se realizar de maneira efetiva. É preciso que os museus sejam bastante objetivos e claros no planejamento e execução das atividades propostas. Para isso, é preciso que, entre outros aspectos, os museus adotem em sua concepção museográfica distintas abordagens sobre uma mesma temática, permitindo assim uma melhor integração por parte do visitante. Dessa forma, percebeu-se que a exposição "Animais Peçonhentos em Rede" cumpriu o seu papel multi-museus, justamente por contar com uma ampla variedade de ferramentas educativas, atendendo assim à pluralidade do seu público visitante.

Geralmente, são os museus universitários que abraçam a tarefa de comunicar o resultado das pesquisas realizadas pela própria universidade. Entre as instituições que, em parceria, promoveram a exposição "Animais Peçonhentos em Rede" apenas o NOAP/UFBA é considerado como um museu universitário. Entretanto, as demais instituições associadas também cumprem similar função por serem centros de pesquisa que produzem e divulgam conhecimento científico sobre animais peçonhentos. Esse tipo de atividade possui caráter inovador 
dentro dos eventos científicos, uma vez que reúne o acervo de diferentes museus de animais peçonhentos em uma exposição aberta. Neste caso, as instituições disponibilizaram seus próprios pesquisadores, técnicos e estudantes para, além de discutirem a temática dentro da academia, dialogar diretamente com o público.

Com o uso do Teatro de Fantoches, os autores sugerem que antes das exposições e demais ações educativas, o público seja recepcionado de maneira lúdica, interativa e divertida, através da apresentação de peças de curta duração. Daí cria-se uma relação mais próxima entre os mediadores e o público e permite que a troca de conhecimentos ocorra com mais motivação, um dos fatores principais não só para o sucesso da aprendizagem, como também na aquisição de novos conhecimentos (DIAS et al., 20I5). As adaptações das peças apresentadas no Teatro de Fantoches foram importantes por acrescentar ao roteiro informações sobre a vida de Vital Brazil, uma vez que nasceu na cidade anfitriã do encontro.A temática dos fantoches enfatizou principalmente sua descoberta sobre a especificidade do soro para os acidentes por animais peçonhentos no Brasil e no mundo.

Assim como o Teatro de Fantoches, a Zooteca e Zookits despertaram bastante o interesse do público infanto-juvenil por apresentar ferramentas atrativas, como jogos e experimentos, correspondendo às expectativas dos autores na inserção deste tipo de atividade, para este público, na composição da exposição. Contudo, é importante salientar que até mesmo os adultos se divertem e aprendem a partir destas experiências lúdicas voltadas para o ensino básico (HUNT, 1973).

A Casa de Vital Brazil, por sua vez, foi o setor da exposição essencial no papel de resgatar a memória do médico homenageado no $2^{\circ}$ EIVB, através da exposição de cartas, fotografias e objetos pessoais.

A utilização de recursos audiovisuais por meio de vídeos, por exemplo, possibilita, através da síntese entre imagem e som, um mecanismo diferente para compreensão do conteúdo (LIMA, 200I). O projeto "Ciência em Movimento" utilizou estes recursos sobre os animais peçonhentos para com os visitantes. Além disso, mediou a comunicação sobre os diferentes meios de produção existentes para com estes animais, como a relação existente entre abelhas e o mel e os cavalos envolvidos na produção dos soros antiofídicos.

É documentado na literatura que a utilização de animais vivos durante as ações educativas sobre os peçonhentos desperta interesse do público (SMANIA-MARQUES; SOUZA; LIRA-DA-SILVA, 2006; MARQUES; LIRA-DA-SILVA, 20I4), o que foi possível observar a partir da coleção viva de serpentes e aracnídeos do Instituto Vital Brazil durante a exposição.

As réplicas do Instituto Butantan chamaram à atenção, em especial, dos participantes do $2^{\circ}$ EIVB (Figura 2 - E). Considerando que os congressistas são em sua maioria pesquisadores e estudantes sobre animais peçonhentos, as réplicas podem ser consideradas como atrativas para este público devido a precisão e riqueza de detalhes, aproximando-os do real. Estes objetos e aparatos interativos foram utilizados na exposição. A questão apontada por Hein (2009) é que, para que sejam eficazes, é necessário que os visitantes gastem neles um tempo considerável e em geral não se compreender essas réplicas em poucos segundos, considerando que nosso objetivo era ter algum impacto sobre seu pensamento dos visitantes. Para resolver isso, disponibilizamos um lugar para sentar e manipular os objetos, com mesa e bancos altos.

O compromisso de divulgar sobre animais peçonhentos, dentro deste 
evento científico, é certamente uma herança de Vital Brazil, pesquisador pioneiro no estudo dos animais peçonhentos brasileiros e fundador de duas das quatro instituições envolvidas na exposição. No início do século XX,Vital Brazil já promovia visitas monitoradas ao Instituto Butantan, intituladas "Fazenda das Cobras" (BRAZIL, I9II; PUORTO, 20I I; PUORTO, 20I2). Tal pioneirismo em educar sobre animais peçonhentos pode ser classificado, hoje, no que se conhece como "ciência cidadã", uma área do conhecimento que busca integrar a pesquisa científica ao público leigo (SOCIENTIZE PROJECT, 20I3).As atividades educativas realizadas durante a exposição, tomando como base as ações pioneiras de Vital Brazil no trato dos animais peçonhentos, aproximou a população de Campanha da prática científica. Esta relação entre o público e a ciência presente durante a visita à exposição, divulgou os objetos educacionais expostos, além de fornecer um retorno à comunidade local sobre a temática principal dos trabaIhos desenvolvidos pelo médico cientista Dr.Vital Brazil.

\section{Considerações Finais}

O esforço conjunto de diversas instituições de ensino e pesquisa em realizar a exposição 'Animais Peçonhentos em Rede' consolidou o papel educativo da RedeVital para o Brasil. O principal desafio neste tipo de atividade ainda consiste, principalmente, na aversão generalizada do público à fauna de animais peçonhentos. Ainda assim, é possível perceber que esta aversão, concomitantemente, também parece estar associada a curiosidade e fascínio.

Apesar da exposição ter atendido à um público diversificado, as diferentes ferramentas educativas utilizadas obtiveram êxito em entreter, comunicar e estabelecer um diálogo sobre os animais peçonhentos e suas interfaces.

\section{Referências}

ANDRADE, Pedro. A museabilidade e a literacia da ciência: consumos, cidadania e cultura. In: ANDRADE, Pedro. (Org.). Museus, públicos e literacia científico-tecnológica. Redes de Comunicação de Significados no Espaço Interdimensional do Museu. Lisboa: Edições Colibri, p. 33-70, 2010.

ARANHA, Caroline Oliveira \& LIRA-DA-SILVA, Rejâne Maria. Um panorama do ensino sobre animais peçonhentos na formação do biólogo em instituições federais de ensino superior no Brasil. In: Livro de Resumos do Encontro Internacional Vital para o Brasil sobre Animais Peçonhentos, Rio de Janeiro, p. 88, 2013. BRAZIL,Vital.A defesa contra o ofidismo. São Paulo: Pocai \& Weiss, I91।.

BRAZIL, Tania Kobler. Rede Vital Para o Brasil - uma experiência em Rede na Ofiologia e Animais peçonhentos. Gazeta Medica da Bahia, v. 82, p. I I0-I I 5, 20 I 2. BRAZIL, Tania Kobler; LIRA-DA-SILVA, Rejâne Maria; GIMENEZ, Anibal Rafael Melgarejo;WERNECK de CASTRO, Antônio Joaquim. Vital Network for Brazil. National Network of Information, Discussion, and Cooperation concerning Venomous Animals. The Journal of Venomous Animals and Toxins Including Tropical Diseases, v. 17, p. 235-236, $201 \mathrm{l}$.

DIAS, Felipe Barbosa; FONSECA, Micheli Ferreira; BARATA, Rita; LOURENÇO,Marta \& LIRA-DA-SILVA, Rejâne Maria. A educação em museus: um intercâmbio Brasil-Portugal com o teatro de fantoches no Museu Nacional de História Natural e da Ciência de Lisboa.Anais do XVI Encontro Nacional de Educação em Ciências. Lisboa: Universidade Nova de Lisboa, p.I3I-I36, 2015. FUNED. Ciência em Movimento. Disponível em: http://www.funed.mg.gov.br/ 
formulario-ciencia-movimento, 20I4.Acesso em: I5 de janeiro de 2017. HEIN, George E. O dilema da Educação Científica: como ensinar quando os visitantes apenas querem aprender. In: MARANDINO, Marta; MORTARA,Adriana; valente, Maria Esther (Orgs.). Museu: lugar do público. Rio de Janeiro: Editora Fiocruz, p. 63-75, 2009.

HUNT, A. Improvisação com adultos. In: BUCKMAN, P. Educação sem escolas. Rio de Janeiro: Livraria Eldorado, 1973.

LIMA,Artemilson Alves de. $O$ uso do vídeo como instrumento didático e educativo em sala de aula. Um estudo de caso do CEFET-RN. Dissertação de Mestrado. Programa de PósGraduação em Engenharia de Produção. Florianópolis: UFSC, 200 I. Disponível em: http://repositorio.ufsc.br/xmlui/handle//23456789/79843 Acesso em: 18 de agosto de 2017.

LIRA-DA-SILVA, Rejâne Maria. Zooamigos. Salvador: Editora da Universidade Federal da Bahia (EDUFBA), 20 I I.

LIRA-DA-SILVA, Rejâne Maria; RABELO, Daniele Silva; SILVA, Luís Fernando Gonçalves da \& LEAL, Marcos Vinícius Cunha. $O$ ensino da zoologia através do teatro de fantoches. In: LIRA-DA-SILVA, Rejâne Maria (Org.). Laboratório do mundo: o jovem e a ciência. Salvador: EDUFBA, p.69-75, 2007.

MARANDINO, Marta. (Org.). Educação em museus: A mediação em foco. São Paulo: FEUSP, 2008.

MARANDINO, Marta. Educação e museus: Da coleção para o público. Revista Jovens Cientistas, ano I, n. 2, p. 34, 2014.

MARQUES, Rodrigo. LIRA-DA-SILVA, Rejâne Maria. Educational actions of Rede de Zoologia Interativa (REDEZOO) about venomous animals: what does the public think? In: Livro de Resumos do $2^{\circ}$ Encontro Internacional Vital para 0 Brasil sobre Animais Peçonhentos, Minas Gerais, p. 46, 2014.

PINTO-COELHO, Daniela. Carlinhos e a Cascavel. Disponível em: http://www. redezoo.ufba.br/teatro/carlinhos-cascavel.pdf. 2010 Acesso em: 29 de março de 2017.

PUORTO, Giuseppe.Vital Brazil e a educação. In:A defesa contra o ofidismo 100 anos depois. Comentários. Niterói: Instituto Vital Brazil, p. 35-39, 201 I.

PUORTO, Giuseppe. Divulgação científica sobre animais peçonhentos no Brasil. Gazeta Médica da Bahia, vol. 82, supl. I, p. 33-39, 2012.

PUORTO, Giuseppe. Arte \& Herpetologia: Replicando Réplicas. Revista Jovens Cientistas. Ano I, n.2, p.20-2I, 2014.

REIS, B.M.; NOVAES, V.P.M.; MACHADO, Cláudio; HAMDAN, Breno. A coleção viva de serpentes da divisão de herpetologia, Instituto Vital Brazil e seu papel na produção, extensão e pesquisa. In: Livro de Resumos do Encontro Nacional sobre Coleções Biológicas e suas Interfaces, p. 52, 2014.

SANTOS, Maria Dulcinéia Sales dos; LIRA-DA-SILVA, Rejâne Maria. Rede de Zoologia Interativa: É possível uma mudança no perfil conceitual de estudantes do ensino médio sobre animais peçonhentos? Gazeta Médica da Bahia, vol. 82, supl. I, p. 40-45, 2012.

SMANIA-MARQUES, Roberta; SOUZA, Jacqueline Silva; LIRA-DA-SILVA, Rejâne Maria. Rede de zoologia interativa - Popularizando e desmistificando os animais peçonhentos. In: LIRADA-SILVA, Rejâne Maria (Org.).A ciência, a arte \& a magia da educação científica. Salvador: Editora da Universidade Federal da Bahia, p. | 2 |-|3|. 2006.

SOCIENTIZE PROJECT. Green Paper on Citizen Science. 2013. Disponível em: http://www.socientize.eu/ Acesso em 19 de agosto de 2017 